\title{
Wronskian Addition Formula and Darboux-Pöschl-Teller Potentials
}

\begin{abstract}
Pierre Gaillard and Vladimir Matveev
Institut de Mathématiques de Bourgogne, UMR 5584 CNRS, Université de Bourgogne, Faculté des Sciences Mirande, 9 Avenue Alain Savary, BP 47870, 21078 Dijon Cedex, France
\end{abstract}

Correspondence should be addressed to Pierre Gaillard; pierre.gaillard@u-bourgogne.fr

Received 30 November 2012; Accepted 25 February 2013

Academic Editor: Alberto Enciso

Copyright (C) 2013 P. Gaillard and V. Matveev. This is an open access article distributed under the Creative Commons Attribution License, which permits unrestricted use, distribution, and reproduction in any medium, provided the original work is properly cited.

For the famous Darboux-Pöschl-Teller equation, we present new wronskian representation both for the potential and the related eigenfunctions. The simplest application of this new formula is the explicit description of dynamics of the DPT potentials and the action of the KdV hierarchy. The key point of the proof is some evaluation formulas for special wronskian determinant.

\section{Introduction}

In 1882 Darboux [1] proved the integrability of the SturmLiouville equation (Darboux also considered and solved an equation which is a generalisation in elliptic functions of (1): see [2]. A hundred years later the integrability of the same elliptic model was obtained by Treibich and Verdier using completely different methods: see $[3,4])$ :

$$
-y^{\prime \prime}+\left(\frac{m(m+1) \alpha^{2}}{\sin ^{2} \alpha x}+\frac{n(n+1) \alpha^{2}}{\cos ^{2} \alpha x}\right) y=\lambda y .
$$

Later in 1933, in the frame of the study of the quantum theory of two atomic molecules, Pöschl and Teller [5] (creator of the hydrogenous bomb) rediscover the hyperbolic version of (1) and proved independently of Darboux the integrability of the equation obtained from (1) by the transformation $\alpha \rightarrow i \alpha$. A lot of authors studied the Schrödinger equation

$$
-\partial_{x}^{2} f+u(x) f=\lambda f,
$$

with the same potentials (mainly called Pöschl-Teller potentials in the literature of quantum mechanics, sometimes called the Nantanzon potentials or Morse-Rosen potentials). In the following, these potentials will be called the Darboux-PöschlTeller potentials or most simply the DPT potentials.

Darboux gave two methods to solve (1) (he considered the case $\alpha=1$ ).
First in exploring the fact the functions defined by

$$
\begin{gathered}
y_{m, n}(x)=\cos ^{n+1}(\alpha x) \sin ^{m+1}(\alpha x), \\
\tilde{y}_{m, n}(x)=\cos ^{-n}(\alpha x) \sin ^{-m}(\alpha x)
\end{gathered}
$$

represent particular solutions of (1) with, respectively, $\lambda=$ $\alpha^{2}(m+n+2)^{2}$ and $\lambda=\alpha^{2}(m+n)^{2}$, precisely using them as generator functions $\varphi$ of the transformation defined by

$$
\partial_{x}-\frac{\varphi_{x}}{\varphi} I
$$

that we call today Darboux transformation; he obtained the general solution of (1) in terms of elementary functions (for $f$ solution of $(2))$ :

$$
y=D_{m} \cdot D_{m-1} \cdots D_{1} f, \quad D_{k}=\partial_{x}-\sigma_{k} I
$$

with

$$
\begin{gathered}
\sigma_{j}=j \alpha \cot \alpha\left(x-x_{0}\right), \quad j \leq m-n, \\
\sigma_{m-n+k}=(m-n+k) \alpha \cot \alpha\left(x-x_{0}\right) \\
\quad-k \alpha \tan \alpha\left(x-x_{0}\right), \quad k=1, \ldots, n .
\end{gathered}
$$

Then taking $\sin ^{2} \alpha x$ as a new independent variable, he reduced (1) to a Gauss hypergeometric equation and expressed 
explicitly the solutions in terms of Gauss hypergeometric functions:

$$
y=\sin ^{m}(\alpha x) \cos ^{n}(\alpha x) g\left(\sin ^{2}(\alpha x)\right),
$$

where $g$ is a solution of Gauss hypergeometric equation:

$$
x(1-x) y^{\prime \prime}+(\gamma-(\delta+\beta+1) x) y^{\prime}-\delta \beta y=0 .
$$

In particular for ${ }_{2} F_{1}$ defined by

$$
{ }_{2} F_{1}(\delta, \beta, \gamma, z)=\sum_{k=0}^{+\infty} \frac{(\delta)_{k}(\beta)_{k}}{(\gamma)_{k}(1)_{k}} z^{k}
$$

with

$$
\begin{gathered}
(a)_{0}=1, \quad(a)_{k}=a(a+1) \cdots(a+k-1), \quad k \neq 0, \\
\gamma=m+\frac{1}{2}, \quad \delta=\frac{m+n}{2}+\frac{1}{2 \alpha} \sqrt{\lambda}, \\
\beta=\frac{m+n}{2}-\frac{1}{2 \alpha} \sqrt{\lambda},
\end{gathered}
$$

$y=\sin ^{m}(\delta x) \cos ^{n}(\alpha x){ }_{2} F_{1}\left(\alpha, \beta, \gamma, \sin ^{2} \alpha x\right)$ is a solution of (1). We present here new representations for the Darboux-Pöschl-Teller potentials and for eigenfunctions of the Schrödinger operators corresponding in terms of wronskians. To explain their nature, we must recall results obtained by Crum [6] in 1955. As usual the wronskian of $m$ functions is defined by the formula

$$
W\left(f_{1}, f_{2}, \ldots, f_{m}\right):=\operatorname{det}\left\|\partial_{x}^{j-1} f_{k}(x)\right\| ; \quad j, k=1, \ldots, m .
$$

We denoted by $\varphi_{j}, f$ some independent solutions of (2) associated, respectively, with $\lambda_{j}$ and $\lambda$; that is,

$$
\begin{gathered}
-\varphi_{j}^{\prime \prime}+u \varphi_{j}=\lambda_{j} \varphi_{j}, \\
-f^{\prime \prime}+u f=\lambda f .
\end{gathered}
$$

We define then the new potential $\widetilde{u}$ and the function $\Psi$ by the formulas

$$
\begin{gathered}
\widetilde{u}=u-2 \partial_{x}^{2}\left[\ln W\left(\varphi_{1}, \ldots, \varphi_{m}\right)\right], \\
\Psi=\frac{W\left(\varphi_{1}, \ldots, \varphi_{m}, f\right)}{W\left(\varphi_{1}, \ldots, \varphi_{m}\right)} .
\end{gathered}
$$

Then we have the following result (Crum's Th. [6]).

Theorem 1. $\Psi$ represents a general solution of (2):

$$
-\partial_{x}^{2} \Psi+\widetilde{u} \Psi=\lambda \Psi,
$$

with $\tilde{u}$ defined by (13), if $f$ is a solution of (2) associated with $u$ and $\lambda$.
Then we can ask the following natural question: how to choose the functions $\varphi_{j}$ solution of (2) in the case $u=0$ so that the potential $\tilde{u}$ defined by (13) is equal to DPT potential

$$
\left(\frac{m(m+1) \alpha^{2}}{\sin ^{2} \alpha x}+\frac{n(n+1) \alpha^{2}}{\cos ^{2} \alpha x}\right) \text {. }
$$

The choice of the introduction of parameter $\alpha$ makes it possible to change from trigonometric model to hyperbolic model and vice versa without any difficulty: it is sufficient for this to change $\alpha$ in $i \alpha$.

We first compute a certain type of wronskians which gives a sort of addition formula.

Then we get a representation of the solutions of (2) in terms of functions $\varphi_{i}$ if we replace in the general solution

$$
\tilde{y}_{m, n}=\frac{W\left(\varphi_{1}, \ldots, \varphi_{m}, f\right)}{W\left(\varphi_{1}, \ldots, \varphi_{m}\right)}
$$

of (2) with

$$
u=-2 \partial_{x}^{2}\left[\ln W\left(\varphi_{1}, \ldots, \varphi_{m}\right)\right], \quad \varphi_{i}^{\prime \prime}=\lambda_{i} \varphi_{i} .
$$

Darboux's formula defined by (5) represents then a direct consequence of (13) and (14) and the formalism of Darboux's transformations methods developed by Matveev [7, 8] (see also [9]).

One of the advantages of this potential representation with wronskians (14) is that it makes it possible to describe the action of the flow of the KdV equation and its hierarchy on these DPT potentials taken as initial data; by replacing the arguments of functions $\varphi_{p}(x)$ by convenient arguments $\varphi_{p}(x+4 \lambda t)$, we get the solutions of the hierarchy of the $\mathrm{KdV}$ equations with DPT initial data without difficulty as in $[7,8]$.

Initial motivation of this paper was the following question. How to select the solutions $f_{j}$ of the free Shrödinger equation $-f_{j}^{\prime \prime}=E_{j} f_{j}$ in order to get the DPT potential from Crum formula (63) with $v=0$. The choice of $E_{j}$ was clear from [10] but at every point $E_{j}$ we have the generic solution $A_{j} \sin \left(\sqrt{E_{j}} x+x_{j}\right)$, where $A_{j}$ plays no role in computing $\partial_{x}^{2} \log W\left(f_{1}, \ldots, f_{m}\right)$. In general, there is no compact expression for the wronskian provided that the phases are chosen arbitrarily. It was also clear that some selection of phases $x_{j}$ leading to DPT potential via (63) should exist but the fact that trivial phases provide the right answer a priori was not obvious and it was necessary to prove (24) (at least modulo constant normalization factor $c_{m n}$ ) in order to confirm it.

This result first appeared in [11], and its abbreviated version makes a part of [12]. Here we give a more detailed and partially new proof of these results. Another different proof was very recently obtained in [13].

We can compare this result with these obtained recently for the discrete version of the Schrodinger equation by the authors [14-16], respectively, called DDPT-I and DDPT-II models. Like in the continuous version where the solutions can be expressed with wronskian determinant, the solutions of these DDPT models can be expressed with the Casorati determinants $[17,18]$. 


\section{Wronskian Addition Formula for Sine Functions}

Let $n$ and $d$ be some nonnegative integers, $m=n+d, \alpha$ and $x_{0}$ be some real parameters, and $\varphi_{p}$ are the functions defined as follows:

$$
\begin{array}{r}
\varphi_{p}(x)=\sin \alpha a_{p}\left(x-x_{0}\right), \quad a_{p}=p, \\
\text { if } d \neq 0,1 \leq p \leq m-n,
\end{array}
$$

$$
a_{j}=a_{j}(m, n)=n-m+2 j, \quad \text { if } n \neq 0, m-n+1 \leq j \leq m .
$$

We use below the standard notation

$$
W\left(f_{1}, f_{2}, \ldots, f_{m}\right):=\operatorname{det}\left\|\partial_{x}^{j-1} f_{k}(x)\right\| ; \quad j, k=1, \ldots, m,
$$

for wronskian determinant of any $m$ functions and the short notation $W_{m n}=W_{m n}\left(\alpha, x, x_{0}\right)=W\left(\varphi_{1}, \ldots, \varphi_{m}\right)$, where $\varphi_{p}$ is defined in (19).

With these notations we have the following formulas (product of factorials in this formulas can be also written as a product of powers):

$$
1 ! \cdot 2 ! \cdots k !=1^{k} \cdot 2^{k-1} \cdot 3^{k-2} \cdots k .
$$

Theorem 2. Consider the following:

$$
\begin{gathered}
W_{m 0}=(-2 \alpha)^{m(m-1) / 2} \prod_{k=0}^{m-1} k ! \cdot \sin ^{m(m+1) / 2} \alpha\left(x-x_{0}\right), \\
W_{m n}=c_{m n} \cdot \sin ^{m(m+1) / 2} \alpha\left(x-x_{0}\right) \cos ^{n(n+1) / 2} \alpha\left(x-x_{0}\right), \\
c_{m n}=(-2 \alpha)^{m(m-1) / 2} \cdot 2^{n^{2}} \prod_{p=0}^{d-1} p ! \prod_{k=1}^{n} \frac{(d+2 k-1) !}{(2 k-1) !}(k-1) !, \\
c_{m m}=(-2 \alpha)^{m(m-1) / 2} \cdot 2^{m^{2}} \prod_{k=1}^{m-1} k !, \quad m \geq 2 .
\end{gathered}
$$

We note $\widehat{W}_{m n}$ to be the determinant obtained from $W_{m n}$ replacing sine functions $\varphi_{j}$ by the sinh functions $g_{j}$ of the same argument:

$$
\begin{gathered}
\widehat{W}_{m n}=W\left(g_{1}, \ldots, g_{m}\right), \\
g_{p}(x)=\sinh \alpha a_{p}\left(x-x_{0}\right), \quad a_{p}=p, \\
\text { if } d \neq 0,1 \leq p \leq m-n ; \\
a_{m-n+j}=m-n+2 j, \quad \text { if } n \neq 0,1 \leq j \leq n .
\end{gathered}
$$

Then the following hyperbolic versions of (23) and (24) hold.

\section{Theorem 3.}

$$
\widehat{W}_{m 0}=(2 \alpha)^{m(m-1) / 2} \prod_{k=0}^{m-1} k ! \cdot \sinh ^{m(m+1) / 2} \alpha\left(x-x_{0}\right),
$$

$$
\begin{aligned}
\widehat{W}_{m n}= & \left|c_{m n}\right| \cdot \sinh ^{m(m+1) / 2} \alpha\left(x-x_{0}\right) \\
& \times \cosh ^{n(n+1) / 2} \alpha\left(x-x_{0}\right), \quad m \geq 2,
\end{aligned}
$$

where $c_{m n}$ is the same as before.

Replacing (19) by the system of functions,

$h_{p}(x)=\cos \alpha a_{p}\left(x-x_{0}\right), \quad a_{p}=p, \quad$ if $p$ is odd,

$h_{p}(x)=\sin \alpha a_{p}\left(x-x_{0}\right), \quad a_{p}=p, \quad$ if $p$ is even,

if $d \neq 0,1 \leq p \leq m-n$,

$a_{m-n+j}=m-n+2 j, \quad$ if $n \neq 0,1 \leq j \leq n$,

$h_{m-n+j}(x)=\cos \alpha a_{m-n+j}\left(x-x_{0}\right), \quad$ if $m-n$ is odd,

$h_{m-n+j}(x)=\sin \alpha a_{m-n+j}\left(x-x_{0}\right), \quad$ if $m-n$ is even;

we get for their wronskian

$$
w_{m n}=W\left(h_{1}, \ldots, h_{m}\right)
$$

the following analogue of (23) and (24).

Theorem 4. Consider the following:

$$
\begin{gathered}
w_{m 0}=(-1)^{(m-1) m^{2} / 2}(2 \alpha)^{m(m-1) / 2} \prod_{k=0}^{m-1} k ! \cdot \cos ^{m(m+1) / 2} \alpha\left(x-x_{0}\right), \\
w_{m n}=(-1)^{(m-1)\left((m-n)^{2}+n\right) / 2}\left|c_{m n}\right| \cdot \cos ^{m(m+1) / 2} \alpha\left(x-x_{0}\right) \\
\quad \times \sin ^{n(n+1) / 2} \alpha\left(x-x_{0}\right), \quad m \geq 2,
\end{gathered}
$$

where $c_{m n}$ has the same meaning as before.

Finally, replacing in the latter formulas sin and cos functions $h_{j}$ by sinh and cosh functions $l_{j}$ of the same arguments and using the notation $\widehat{w}_{m n}$ for their wronskian we get the formulas

$\widehat{w}_{m n}=W\left(l_{1} \ldots, l_{m}\right)$,

$l_{p}(x)=\cosh \alpha a_{p}\left(x-x_{0}\right), \quad a_{p}=p, \quad$ if $p$ is odd,

$l_{p}(x)=\sinh \alpha a_{p}\left(x-x_{0}\right), \quad a_{p}=p, \quad$ if $p$ is even,

if $d \neq 0,1 \leq p \leq m-n$,

$a_{m-n+j}=m-n+2 j, \quad$ if $n \neq 0,1 \leq j \leq n$,

$l_{m-n+j}(x)=\cosh \alpha a_{m-n+j}\left(x-x_{0}\right), \quad$ if $m-n$ is odd,

$l_{m-n+j}(x)=\sinh \alpha a_{m-n+j}\left(x-x_{0}\right), \quad$ if $m-n$ is even.

Theorem 5. Consider the following:

$$
\widehat{w}_{m 0}=(2 \alpha)^{m(m-1) / 2} \prod_{k=0}^{m-1} k ! \cdot \cosh ^{m(m+1) / 2} \alpha\left(x-x_{0}\right),
$$




$$
\begin{aligned}
\widehat{w}_{m n}= & \left|c_{m n}\right| \cdot \cosh ^{m(m+1) / 2} \alpha\left(x-x_{0}\right) \\
& \times \sinh ^{n(n+1) / 2} \alpha\left(x-x_{0}\right), \quad m \geq 2 .
\end{aligned}
$$

Proof. All the results given in (24), (23), (27), (30), (33), and (32) can be proved in the same way; we present, for example, the case ((24), (23)) where the functions are defined by (19). Then all the others results can be obtained by changes of variables; precisely, wet get (27) from ((24), (23)) by the change $\left(x-x_{0}\right) \rightarrow i\left(x-x_{0}\right)$, (30) from ((24), (23)) by the change $\left(x-x_{0}\right) \rightarrow(\pi / 2 \alpha)+\left(x-x_{0}\right)$, and ((33), (32)) from $((24),(23))$ by the change $\left(x-x_{0}\right) \rightarrow(\pi / 2 \alpha)+i\left(x-x_{0}\right)$. It is sufficient to prove the first result $((24),(23))$.

In the following, we use the notation $q=e^{i \alpha}$. We define the functions $f_{j}$ by the formula

$$
f_{j}=\sin (\alpha j x), \quad \text { with } j \in \mathbb{Z} .
$$

Then it is easy to see that

$$
W_{m n}=W\left(f_{1}, f_{2}, \ldots, f_{m-n}, f_{m-n+2}, f_{m-n+4}, \ldots, f_{m+n}\right) .
$$

We define the sequence of wronskians $W_{m-k, n}$ of order $m-k$ by the formulas

$$
\begin{gathered}
W_{m-1, n}=W\left(f_{1}, f_{2}, \ldots, f_{m-n-1}, f_{m-n+1}, f_{m-n+3}, \ldots, f_{m+n-1}\right) \\
W_{m-k, n}=W\left(f_{1}, f_{2}, \ldots, f_{m-n-k}, f_{m-n-k+2},\right. \\
\left.f_{m-n-k+4}, \ldots, f_{m-k+n}\right) \\
W_{n, n}=W\left(f_{2}, f_{4}, \ldots, f_{2 n}\right) .
\end{gathered}
$$

It is obvious that $W_{n n}(\alpha)=W_{n 0}(2 \alpha)$. We consider the functions equal to $\varphi_{j}$ defined in (19).

The structure of the proof of the first result $((24),(23))$ is the following.

We start with the proof of (23). Next we establish the recursion relation for each $k$ such that $0 \leq k \leq m-n-1$ :

$$
\begin{aligned}
W_{m-k, n}= & f_{1}^{m-k}(-2 \alpha)^{m-k-1}(m-k-1-n) ! \\
& \times \prod_{j=1}^{n}(m-k-1-n+2 j) \cdot W_{m-k-1, n} .
\end{aligned}
$$

For brevity, we replace $x-x_{0}$ by $x$ in the following.

We calculate the wronskian $W_{n n}=W\left(f_{2}, \ldots, f_{2 n}\right)$.

For this, we use the expansion

$$
\begin{aligned}
\sin p x & =\sin x \sum_{k=0}^{[(p-1) / 2]}(-1)^{k} C_{p-k-1}^{k} \cdot(2 \cos x)^{p-1-2 k} \\
& =\sin x\left[(2 \cos x)^{p-1}-(p-2)(2 \cos x)^{p-3}+\cdots\right] .
\end{aligned}
$$

Therefore $W_{n n}$ can be reduced to

$$
\begin{aligned}
& W_{n n} \\
& \quad=W\left(\sin 2 \alpha x, 2 \sin 2 \alpha x \cos 2 \alpha x, \ldots, \sin 2 \alpha x(\cos 2 \alpha x)^{n-1}\right),
\end{aligned}
$$

by replacing each term in the expansion of $\sin 2 p \alpha x$, formula (38) by their first terms, that is, $\sin 2 \alpha x(2 \cos 2 \alpha x)^{p-1}, 2 \leq$ $p \leq n$.

We can also factorize in each column $p$ the term $2^{p-1}$ for $2 \leq p \leq n$, so we can drop out the factor $2^{n(n-1) / 2} ; W_{n n}$ can be written as

$$
\begin{aligned}
W_{n n}= & 2^{n(n-1) / 2} W \\
& \times\left(\sin 2 \alpha x, \sin 2 \alpha x \cos 2 \alpha x, \ldots, \sin 2 \alpha x(\cos 2 \alpha x)^{n-1}\right) .
\end{aligned}
$$

Then we use the two following statements.

Proposition 6. For any smooth functions $f, \varphi_{1}, \ldots, \varphi_{n}$, the wronskian $W\left(f \cdot \varphi_{1}, \ldots, f \cdot \varphi_{n}\right)$ verify

$$
W\left(f \cdot \varphi_{1}, \ldots, f \cdot \varphi_{n}\right)=(f(x))^{n} W\left(\varphi_{1}, \ldots, \varphi_{n}\right),
$$

first proved by Frobenius [19]. The second one is a well-known formula of change of independent variable in a wronskian.

Proposition 7. For $t=\varphi(x)$ and $\tilde{f}_{j}$ defined by $f_{j}(x)=\tilde{f}_{j}(t)$ :

$$
W\left(f_{1}, \ldots, f_{n}\right)=\left(\varphi^{\prime}(x)\right)^{n(n-1) / 2} \cdot W\left(\tilde{f}_{1}, \tilde{f}_{2}, \ldots, \tilde{f}_{n}\right) .
$$

Then we can factorize $(\sin (2 \alpha x))^{n}$ in the preceding determinant $W_{n}$ to get

$$
W_{n n}=2^{n(n-1) / 2}(\sin (2 \alpha x))^{n} W\left(1, \cos 2 \alpha x, \ldots,(\cos 2 \alpha x)^{n-1}\right) .
$$

Here, we make the change of variable defined by $t=\varphi(x)=$ $\cos 2 \alpha x$. This leads us to appearance of the extra factor $(-2 \alpha \sin 2 \alpha x)^{n(n-1) / 2}$. Therefore the wronskian $W_{n}$ can be written as

$$
\begin{aligned}
W_{n n}= & 2^{n(n-1) / 2}(-2 \alpha \sin 2 \alpha x)^{n(n-1) / 2} \\
& \times(\sin (2 \alpha x))^{n} W\left(1, t, \ldots, t^{n-1}\right) .
\end{aligned}
$$

The wronskian $W\left(1, t, \ldots, t^{n-1}\right)$ has the upper triangular form and thus is equal to the product of its diagonal elements; in other words,

$$
W\left(1, t, \ldots, t^{n-1}\right)=\prod_{j=1}^{n-1} j !
$$

So $W_{n}$ can be written as

$$
W_{n n}=2^{n(n-1)}(-\alpha)^{n(n-1) / 2} \prod_{j=1}^{n-1} j ! \cdot(\sin 2 \alpha x)^{n(n+1) / 2} .
$$

Expanding $\sin 2 \alpha x$, we get finally

$$
\begin{aligned}
W_{n n}= & 2^{n(n-1)+(n(n+1) / 2)}(-\alpha)^{n(n-1) / 2} \\
& \times \prod_{j=1}^{n-1} j ! \cdot(\sin \alpha x)^{n(n+1) / 2}(\cos \alpha x)^{n(n+1) / 2} .
\end{aligned}
$$


In a second step, we establish a recursion relation between the wronskians $W_{m n}$ and $W_{m-1, n}$ (here $\left.m>n\right)$.

We can factorize $\varphi_{1}^{m}$ in $W_{m n}$ to get

$$
W_{m n}=\varphi_{1}^{m} \cdot\left|\begin{array}{cccc}
1 & \frac{\varphi_{2}}{\varphi_{1}} & \cdots & \frac{\varphi_{m}}{\varphi_{1}} \\
\frac{\varphi_{1}^{(1)}}{\varphi_{1}} & \frac{\varphi_{2}^{(1)}}{\varphi_{1}} & \cdots & \frac{\varphi_{m}^{(1)}}{\varphi_{1}} \\
\vdots & \vdots & \vdots & \vdots \\
\frac{\varphi_{1}^{(m-1)}}{\varphi_{1}} & \frac{\varphi_{2}^{(m-1)}}{\varphi_{1}} & \cdots & \frac{\varphi_{m}^{(m-1)}}{\varphi_{1}}
\end{array}\right| .
$$

It can be written as

$$
W_{m n}=\varphi_{1}^{m} \cdot\left|\begin{array}{cccc}
\left(\frac{\varphi_{2}}{\varphi_{1}}\right)^{(1)} & \left(\frac{\varphi_{3}}{\varphi_{1}}\right)^{(1)} & \cdots & \left(\frac{\varphi_{m}}{\varphi_{1}}\right)^{(1)} \\
\vdots & \vdots & \vdots & \vdots \\
\left(\frac{\varphi_{2}}{\varphi_{1}}\right)^{(m-1)} & \left(\frac{\varphi_{3}}{\varphi_{1}}\right)^{(m-1)} & \cdots & \left(\frac{\varphi_{m}}{\varphi_{1}}\right)^{(m-1)}
\end{array}\right| .
$$

In a shortened way we get the recursion relation

$$
W_{m n}=\varphi_{1}^{m} \cdot W\left(\left(\frac{\varphi_{2}}{\varphi_{1}}\right)^{(1)}, \ldots,\left(\frac{\varphi_{m}}{\varphi_{1}}\right)^{(1)}\right) .
$$

We can evaluate the quotient $\varphi_{p} / \varphi_{1}$ in function of $q$; namely,

$$
\begin{aligned}
\frac{\varphi_{p}}{\varphi_{1}} & =\frac{q^{a_{p}(x)}-q^{-a_{p}(x)}}{q^{(x)}-q^{-(x)}} \\
& =q^{\left(a_{p}-1\right)(x)}+q^{\left(a_{p}-3\right)(x)}+\cdots+q^{-\left(a_{p}-1\right)(x)} .
\end{aligned}
$$

So the $j$ th row of the column $p$ of the determinant $W\left(\left(\varphi_{2} / \varphi_{1}\right)^{(1)}, \ldots,\left(\varphi_{m} / \varphi_{1}\right)^{(1)}\right)$ can be reduced to $\left(q^{\left(a_{p}-1\right)(x)}+\right.$ $\left.q^{-\left(a_{p}-1\right)(x)}\right)^{(j+1)}$ because other terms are combinations of the preceding columns, except for $p=1$ or $p=2$; in this case the constant terms are eliminated by the derivations (e.g., for $a_{3}=3,\left(\varphi_{3} / \varphi_{1}\right)=q^{2 x}+1+q^{-2 x}$, and the derivations eliminate the constant term).

Then each term $\left(\varphi_{p} / \varphi_{1}\right)^{(1)}$ in the determinant $W\left(\left(\varphi_{2} / \varphi_{1}\right)^{(1)}, \ldots,\left(\varphi_{m} / \varphi_{1}\right)^{(1)}\right)$ can be replaced by $\left(q^{\left(a_{p}-1\right)(x)}+\right.$ $\left.q^{-\left(a_{p}-1\right)(x)}\right)^{(1)}$, which can be rewritten as $\alpha i\left(a_{p}-1\right)\left(q^{\left(a_{p}-1\right)(x)}-\right.$ $\left.q^{-\left(a_{p}-1\right)(x)}\right)=-2 \alpha\left(a_{p}-1\right) \sin \left(\left(a_{p}-1\right) x\right)$ for $2 \leq p \leq m$.

Let $\Phi_{p}$ be $\varphi_{p} / \varphi_{1}$. It is obvious that $a_{p}(m, n)-1=$ $a_{p-1}(m-1, n)$, for $2 \leq p \leq m$. So the sequence $\left(\Phi_{2}, \ldots\right.$, $\left.\Phi_{m-n}, \Phi_{m-n+1}, \ldots, \Phi_{m}\right)$ can be replaced by $\left(f_{1}, \ldots f_{m-n-1}\right.$, $\left.f_{m-n+1}, \ldots, f_{m+n-1}\right)$. We get the equality

$$
\begin{aligned}
W_{m n}= & f_{1}^{m} W \\
& \times\left(-2 \alpha f_{1}, \ldots,-2 \alpha(m-n-1) f_{m-n-1},\right. \\
& \left.\quad-2 \alpha(m-n+1) f_{m-n+1}, \ldots,-2 \alpha(m+n-1) f_{m+n-1}\right) .
\end{aligned}
$$

We can drop out the constants appearing in each column to get

$$
\begin{aligned}
W_{m n}= & f_{1}^{m}(-2 \alpha)^{m-1}(m-1-n) ! \\
& \times \prod_{j=1}^{n}(m-1-n+2 j) \cdot W_{m-1, n} .
\end{aligned}
$$

We can make these transformations for each integer $k$ such that $0 \leq k \leq m-n-1$, so we get the recursion relation

$$
\begin{aligned}
W_{m-k, n}= & f_{1}^{m-k}(-2 \alpha)^{m-k-1}(m-k-1-n) ! \\
& \times \prod_{j=1}^{n}(m-k-1-n+2 j) \cdot W_{m-k-1, n} .
\end{aligned}
$$

We apply the recursion relation (54) $(m-n)$ times and we get the expression

$$
\begin{aligned}
W_{m n}= & \sin \alpha(x)^{(m-n)(m+n+1) / 2}(-2 \alpha)^{(m-n)(m+n-1) / 2} \\
& \cdot \prod_{j=0}^{m-n-1} j ! \prod_{k=1}^{m-n} \prod_{j=1}^{n}(k+2 j-1) \cdot W_{n, n} .
\end{aligned}
$$

To compute $W_{m n}$ it is now sufficient to replace $W_{n n}$ given by (47) in $W_{m, n}$ defined by (55). So we get

$$
W_{m n}=(-1)^{m(m-1) / 2} 2^{((m(m-1) / 2)+(n(n+1) / 2)+(n(n-1) / 2))} \alpha^{m(m-1) / 2}
$$

$$
\begin{aligned}
& \cdot \prod_{j=1}^{n-1} j ! \prod_{j=1}^{m-n-1} j ! \prod_{k=1}^{m-n} \prod_{j=1}^{n}(k+2 j-1) ! \\
& \cdot(\sin \alpha x)^{m+\cdots+n+1+(n(n+1) / 2)}(\cos \alpha x)^{n(n+1) / 2} .
\end{aligned}
$$

We can rearrange these terms and get

$$
\begin{aligned}
W_{m n}= & (-2 \alpha)^{m(m-1) / 2} 2^{n^{2}} \cdot \prod_{j=1}^{n-1} j ! \prod_{j=1}^{m-n-1} j ! \prod_{k=1}^{m-n} \prod_{j=1}^{n}(k+2 j-1) ! \\
& \cdot(\sin \alpha x)^{m(m+1) / 2}(\cos \alpha x)^{n(n+1) / 2}
\end{aligned}
$$

It is easy to verify the identity

$$
\prod_{k=1}^{m-n} \prod_{j=1}^{n}(k+2 j-1) !=\prod_{j=1}^{n} \frac{(m-n+2 j-1) !}{(2 j-1) !} .
$$

Finally, we get

$$
\begin{aligned}
W_{m n}= & (-2 \alpha)^{m(m-1) / 2} 2^{n^{2}} \cdot \prod_{j=1}^{m-n-1} j ! \prod_{j=1}^{n} \frac{(m-n+2 j-1) !}{(2 j-1) !} \\
& \times(j-1) ! \cdot(\sin \alpha x)^{m(m+1) / 2}(\cos \alpha x)^{n(n+1) / 2} .
\end{aligned}
$$

Taking into account the notation $d=m-n$, we recover exactly the formula (24). 
In the case where $n=0, W_{m 0}$ can be computed with the obvious relation $W_{n 0}(2 \alpha)=W_{n n}(\alpha)$. So we have

$$
W_{m 0}=(-2 \alpha)^{m(m-1) / 2} \prod_{j=1}^{m-1} j !(\sin \alpha x)^{m(m+1) / 2} \text {. }
$$

Thus, one gets the results of Theorem 2, and consequently those of Theorems 3, 4 and 5 .

Example 8. The following example of wronskian $W_{m n}$ illustrates well the behavior of the coefficients $c_{m n}$ :

$$
\begin{aligned}
& W(\sin x, \sin 3 x, \sin 5 x, \sin 7 x, \sin 9 x) \\
& \quad=2^{35} \cdot 9 \cos ^{10} x \sin ^{15} x \\
& =309237645312 \cos ^{10} x \sin ^{15} x
\end{aligned}
$$

Formulas (24) represent a very strong reduction with respect to combinatorial definition of the related determinant representing a trigonometric polynomial containing $m$ ! terms. It is reduced by (24) to a single monomial term.

\section{Applications of the Addition Formula}

3.1. Wronskian Representation of the DPT Potentials and KdV Equation. Below we need the following two propositions.

Let $v(x)$ be any potential and $f_{1}\left(x, E_{1}\right), f_{2}\left(x, E_{2}\right)$, $\ldots, f_{m}\left(x, E_{m}\right)$ be any solutions of the $1 \mathrm{D}$ Schrödinger (Sturm-Liouville) equation

$$
-f^{\prime \prime}+v(x) f=E f
$$

corresponding to $E=E_{1}, E_{2}, \ldots, E_{m}$.

Proposition 9. The solution $\psi$ of the Schrödinger equation

$$
-\psi^{\prime \prime}+u \psi=E \psi, \quad u=v-2 \partial_{x}^{2} \log W\left(f_{1}, f_{2}, \ldots, f_{m}\right)
$$

is given by the formula

$$
\psi(x, E)=\frac{W\left(f_{1}, f_{2}, \ldots, f_{m}, f(x)\right)}{W\left(f_{1}, f_{2}, \ldots, f_{m}\right)} .
$$

In the case $m=1$, this proposition was found and proved by Darboux [10], and in general case by Crum [6]. Remarkably, their result can be extended to the partial differential equations and their nonabelian and lattice versions, [7] which finds many applications in modern theory of solitons $[8,9]$. Below we need this extension only for the particular case of the scalar evolution PDE of the following form:

$$
\partial_{t} f=\sum_{j=0}^{N} v_{m}(x, t) \partial_{x}^{j} f(x, t)
$$

Let $f_{1}, f_{2}, \ldots, f_{m}$ be any $m$ linearly independent solutions of (65) different from $f$. Then the following statement holds.
Proposition 10 (see $[8,9]$ for the proof and further details). The RHS of (64) satisfy the following PDE:

$$
\partial_{t} \psi=\sum_{j=0}^{N} u_{m}(x, t) \partial_{x}^{j} \psi(x, t)
$$

where the coefficients $u_{m}(x, t)$ can be explicitly written in terms of $v_{j}$ and $f_{k}(x, t)$. In particular, assuming that $v_{N}=$ const and $v_{N-1}=0$ we obtain the following formulas for $u_{N}, u_{N-1}, u_{N-2}$ :

$$
\begin{gathered}
u_{N}=v_{N}, \quad u_{N-1}=v_{N-1}=0, \\
u_{N-2}=v_{N-2}+N v_{N} \partial_{x}^{2} \log W\left(f_{1}, f_{2}, \ldots, f_{m}\right) .
\end{gathered}
$$

The RHS of (64) represents the action of the differential operator of order $m$ on the function $f$ :

$$
\psi=D(m) f:=\left(\partial_{x}^{m}+\sum_{k=0}^{m-1} g_{k}(x, t) \partial_{x}^{k}\right) f .
$$

$D(m)$ can be also represented in a form of a product of the first-order differential operators in many different ways. Let $J=\left(j_{1}, j_{2}, \ldots, j_{m}\right)$ be any permutation of the numbers $(1,2, \ldots, m)$. To each permutation corresponds the following factorization of $D(m)$ :

$$
\begin{gathered}
D(m)=D_{j_{m}} \cdot D_{j_{m-1}} \cdots D_{j_{1}}, \quad D_{j_{k}}=\partial_{x}-\sigma_{j_{k}}, \\
\sigma_{j_{k}}:=\partial_{x} \log \frac{W\left(f_{j_{1}}, f_{j_{2}}, \ldots, f_{j_{k}}\right)}{W\left(f_{j_{1}}, f_{j_{2}}, \ldots, f_{j_{k-1}}\right)} .
\end{gathered}
$$

Assume now that $J=(1,2, \ldots, m), v=0$, and $f_{j}=\varphi_{j}$, where $\varphi_{j}$ are defined in (19). Combining formula (24) with Proposition 9 we get the following result. The function $\psi$ corresponding to the case $f_{j}=\varphi_{j}, v=0$ solves the Schrödinger equation with singular periodic potential $U_{m, n}$ :

$$
-\psi^{\prime \prime}+U_{m, n}(x) \psi=E \psi,
$$

where

$$
\begin{aligned}
U_{m, n} & =-2 \partial_{x}^{2} \log W_{m n} \\
& =\frac{\alpha^{2} m(m+1)}{\sin ^{2} \alpha(x)}+\frac{\alpha^{2} n(n+1)}{\cos ^{2} \alpha(x)}, \quad m>n .
\end{aligned}
$$

Replacing $W_{m n}$ by $\widehat{W}_{m n}$ we obtain the potential $\widetilde{U}_{m, n}$ exponentially decreasing at infinity:

$$
\begin{aligned}
\widetilde{U}_{m, n} & =-2 \partial_{x}^{2} \log \widehat{W}_{m n} \\
& =\frac{\alpha^{2} m(m+1)}{\sinh ^{2} \alpha(x)}-\frac{\alpha^{2} n(n+1)}{\cosh ^{2} \alpha(x)}, \quad m>n .
\end{aligned}
$$

General solution of the related Shrödinger equation (71) is obtained from (64) by choosing $f_{j}=g_{j}$. Quite similarly we have

$$
u_{m n}:=-2 \partial_{x}^{2} \log w_{m n}=\frac{\alpha^{2} m(m+1)}{\cos ^{2} \alpha\left(x-x_{0}\right)}+\frac{\alpha^{2} n(n+1)}{\sin ^{2} \alpha\left(x-x_{0}\right)},
$$


where $w_{m n}$ was defined in (2) and the solution $\psi(x, E)$ of the related Schrödinger equation can be obtained by choosing $f_{j}=$ $h_{j}$ in (64).

It is clear as well that

$$
\begin{aligned}
\widehat{u}_{m n}:= & -2 \partial_{x}^{2} \log \widehat{w}_{m n} \\
= & -\frac{\alpha^{2} m(m+1)}{\cosh ^{2} \alpha\left(x-x_{0}\right)} \\
& +\frac{\alpha^{2} n(n+1)}{\sinh ^{2} \alpha\left(x-x_{0}\right)}, \quad m>n,
\end{aligned}
$$

and the solutions of the related Schrödinger equation are obtained from (64) by choosing $f_{j}=l_{j}$. In the case $n=0$, $\widehat{u}_{m n}$ is a nonsingular reflectionless potential and the discrete negative eigenvalues of the related Schrödinger operator on the real line are $E_{j}=-\alpha^{2} j^{2}$.

The integrability of (71) with potential (72) with $\alpha=$ $1, x_{0}=0$ was the discovered first time by Darboux in 1882 and rediscovered for its hyperbolic version (73) by Pöschl and Teller in 1933. Therefore, it is reasonable to call (72), (73), (74), and (75) the Darboux-Pöschl-Teller or shortly DPT potentials.

The solution of (71) found by Darboux [10] corresponds to particular factorization (70) with $J=(1,2, \ldots, m)$; that is, the related function $\psi$ can be written as

$$
\psi=D_{m} \cdot D_{m-1} \cdots D_{1} f, \quad D_{k}=\partial_{x}-\sigma_{k} .
$$

The related functions $\sigma_{k}$ now can be computed combining (24) and (70), where, of course, the constant factor in the RHS of (24) can be ignored:

$$
\begin{aligned}
\sigma_{j}= & \alpha j \cot \alpha\left(x-x_{0}\right), \quad j \leq m-n, \\
\sigma_{m-n+k}= & (m-n+k) \alpha \cot \alpha\left(x-x_{0}\right) \\
& -k \alpha \tan \alpha\left(x-x_{0}\right), \quad k=1, \ldots, n .
\end{aligned}
$$

Original derivation [10] by Darboux of (77) was based on the observation that the functions $\sin ^{m}\left(x-x_{0}\right) \cos ^{n} \alpha\left(x-x_{0}\right)$ represent the particular solutions of the Schrödinger equation (71) with potential $U_{m-1, n-1}$ corresponding to $E=\alpha^{2}(m+n)^{2}$ and was not using general Crum's formulas as we did.

The advantage of the Crum representation for the DPT potentials is that it allows to describe immediately the action of $\mathrm{KdV}$ and higher flows of the KdV hierarchy on those potentials taken as initial data by using Proposition 10. The $j$ th equation of the KdV hierarchy can be obtained as a compatibility condition of (62) and the following evolution equation:

$$
\begin{aligned}
\partial_{t_{j}} f= & c_{j} \partial_{x}^{2 j+1} f-\frac{(2 j+1) c_{j}}{2} v\left(x, t_{j}\right) \partial_{x}^{2 j-1} f \\
& +\sum_{p=0}^{2 j-2} v_{p}\left(x, t_{j}\right) \partial_{x}^{p} f .
\end{aligned}
$$

In particular for $j=1, c_{j}=-4$, and $v_{0}=3 v_{x}$ compatibility of (62) and (78) implies that $v$ satisfies the KdV equation

$$
v_{t}=6 v v_{x}-v_{x x x} .
$$

In general the choice of $c_{j}$ is arbitrary: it fixes normalization of the KdV hierarchy. Now according to Proposition 10 for any given solution $v\left(x, t_{j}\right)$ of $j$ th $\mathrm{KdV}$ equation the sequence $f_{1}, \ldots, f_{m}$ of the common solutions of (62) and (78) generates the new solution $u\left(x, t_{j}\right)$ of the same equation:

$$
u\left(x, t_{j}\right)=v\left(x, t_{j}\right)-2 \partial_{x}^{2} \log W\left(f_{1}, f_{2}, \ldots, f_{m}\right) .
$$

In particular, in the case

$$
\begin{gathered}
v=0, \\
f_{p}(x, t)=\sin \left[\alpha p\left(x-x_{0}\right)+(-1)^{j} c_{j} \alpha^{2 j+1} p^{2 j+1} t_{j}\right], \\
p=1, \ldots, d, d=m-n, \\
=\sin \left[\alpha(d+2 l)\left(x-x_{0}\right)+(-1)^{j} c_{j} \alpha^{2 j+1}(d+2 l)^{2 j+1} t_{j}\right], \\
l=1, \ldots, n, m \geq n ;
\end{gathered}
$$$$
f_{d+l}(x, t)
$$

the RHS of (80) is the solution of the $j$ th KdV equation with the initial data equal to the DPT potential (72). Therefore, we obtain a very simple description of the isospectral deformations of the DPT potentials generated by the KdV hierarchy. In particular the solution of (79) with the initial condition (72) is given by the formula (80) with $v=0$ and

$$
\begin{array}{r}
f_{p}(x, t)=\sin \left(\alpha p\left(x-x_{0}\right)+4 \alpha^{3} p^{3} t\right), \\
p=1, \ldots, d, d=m-n, \\
f_{d+l}(x, t)=\sin \left[\alpha(d+2 l)\left(x-x_{0}\right)+4 \alpha^{3}(d+2 l)^{3} t\right], \\
l=1, \ldots, n, m \geq n .
\end{array}
$$

Similar formulas for the action of KdV and higher KdV flows on the potentials (74), (73), and (75) are also obvious from the previous discussion. For instance, the solutions of the KdV equation (79) with the initial condition (75), with $v=0$, is given by the formula

$$
v(x, t)=-2 \partial_{x}^{2} \log W\left(L_{1}, \ldots, L_{m}\right),
$$

where the functions $L_{j}(x, t)$ are defined as follows:

$$
\begin{array}{r}
L_{p}(x, t):=\cosh \alpha a_{p}\left(x-x_{0}-4 \alpha^{2} a_{p}^{2} t\right), \quad a_{p}=p, \\
\text { if } p \text { is odd, } \\
L_{p}(x, t):=\sinh \alpha a_{p}\left(x-x_{0}-4 \alpha^{2} a_{p}^{2} t\right), \quad a_{p}=p, \\
\text { if } p \text { is even, }
\end{array}
$$

if $d \neq 01 \leq p \leq m-n$, 


$$
\begin{array}{r}
a_{m-n+j}=m-n+2 j, \quad \text { if } n \neq 0, j=1, \ldots, n, \\
L_{m-n+j}(x, t):=\cosh \alpha a_{m-n+j}\left(x-x_{0}-4 \alpha^{2} a_{m-n+j}^{2} t\right), \\
\text { if } m-n \text { is odd, } \\
L_{m-n+j}(x):=\sinh \alpha a_{m-n+j}\left(x-x_{0}-4 \alpha^{2} a_{m-n+j}^{2} t\right), \\
\text { if } m-n \text { is even. }
\end{array}
$$

3.2. Differential Identities. Formulas (23) and (24) lead to some nontrivial identities. Let $\widehat{D}$ be a differential operator defined by the formula

$$
(\widehat{D} f)(x)=\left.\left(\prod_{l=2}^{m} \partial_{x_{l}}^{l-1}\right) f\left(x_{1}, x_{2}, \ldots, x_{m}\right)\right|_{x_{1}=x_{2}=\cdots=x_{m}=x} .
$$

Let $D_{m}$ be the following determinant: $D_{m}=\operatorname{det} a_{j, k}, a_{j, k}=$ $\sin j x_{k}$, with $1 \leq j, k \leq m$.

Clearly, we have

$$
\begin{aligned}
\widehat{D} D_{m} & =\operatorname{det} \partial_{x}^{j-1} \sin k x \\
& =W(\sin x, \sin 2 x, \ldots, \sin m x)=W_{m 0} .
\end{aligned}
$$

The determinant $D_{m}$ can be easily computed and further obvious reduction to a Vandermonde determinant (the same expression (up to a different constant normalization factor) as the RHS of (33) recently appeared [20, (Th. A, Th. 5.1)] as a density of probability measure describing the asymptotic distribution of Frobenius roots on the $m$-dimensional abelian varieties over finite fields $F_{\alpha}$ when $\alpha \rightarrow \infty$ ):

$$
D_{m}=2^{m(m-1) / 2} \prod_{k=1}^{m} \sin x_{k} \prod_{m \geq k>j \geq 1}\left(\cos x_{k}-\cos x_{j}\right) .
$$

Now, substituting this expression with $D_{m}$ in (86) and taking into account the formula (24) for $W_{m 0}$, we get the following identity:

$$
\begin{aligned}
& \left(\prod_{l=2}^{m} \partial_{x_{l}}^{l-1}\right)\left[\left(\prod_{k=1}^{m} \sin x_{k}\right)\right. \\
& \left.\cdot\left(\prod_{m \geq k>j \geq 1}\left(\cos x_{j}-\cos x_{k}\right)\right)\right]\left.\right|_{x_{1}=x_{2}=\cdots=x_{m}=x} \\
& =\prod_{k=1}^{m-1} k ! \cdot \sin ^{m(m+1) / 2} x, \quad m \geq 2
\end{aligned}
$$

It is obvious that the wronskian

$$
\begin{gathered}
\widetilde{W}_{m n}:=W(1, \cos x, \cos 2 x, \ldots, \cos d x, \cos (d+2) x, \\
\cos (d+4) x, \ldots, \cos (d+2 n) x)
\end{gathered}
$$

is proportional to $W_{m n}$ :

$$
\begin{gathered}
\widetilde{W}_{m n}=(-1)^{m}(d-1) ! \prod_{k=0}^{n}(d+2 k) \cdot W_{m n}, \\
\widetilde{W}_{m 0}=(-1)^{m} m ! \cdot W_{m 0} .
\end{gathered}
$$

This follows immediately from the expansion of $\widetilde{W}$ by the elements of its first column. Assuming that $n=0$, and introducing the determinant,

$$
\begin{gathered}
G_{m}=\operatorname{det} B, \quad B_{j k}=\cos j x_{k}, \\
j, k=0,1, \ldots m, \text { with } x_{0}=x ;
\end{gathered}
$$

we obviously have the following formula:

$$
\widetilde{W}_{m 0}=\left.\left(\prod_{j=1}^{m} \partial_{x_{j}}^{j}\right) G_{m}\right|_{x_{0}=x_{1}=x_{2}=\cdots=x_{m}=x} .
$$

From the other side, $G_{m}$ can be calculated explicitly and the reduction to a Vandermonde determinant is obvious:

$$
G_{m}=2^{m(m-1) / 2} \prod_{m \geq j>k \geq 0}\left(\cos x_{j}-\cos x_{k}\right) .
$$

Comparing these two formulas with explicit expression for $\widetilde{W}_{m 0}$, following immediately from (90), we obtain the following identity:

$$
\begin{aligned}
& \left.\left(\prod_{l=1}^{m} \partial_{x_{l}}^{l}\right) \cdot\left(\prod_{m \geq j>k \geq 0}\left(\cos x_{k}-\cos x_{j}\right)\right)\right|_{x_{0}=x_{1}=x_{2}=\cdots=x_{m}=x} \\
& \quad=\left(\prod_{k=1}^{m} k !\right) \cdot \sin ^{m(m+1) / 2} x, \quad m \geq 2 .
\end{aligned}
$$

Similar but more complicated identities might be obtained along the same lines, comparing (24) with explicit expression for the determinant $\operatorname{det} B, B_{p j}=\varphi_{p}\left(x_{j}\right), j, p=1, \ldots, m$, where the functions $\varphi_{p}(x)$ were defined in (19). For instance for $n=1, \alpha=1$, and $x_{0}=0$ this leads to the following identity:

$$
\begin{gathered}
\left(\prod_{l=2}^{m} \partial_{x_{l}}^{l-1}\right)\left[\left(\prod_{k=1}^{m} \sin x_{k}\right) \cdot\left(\sum_{i=1}^{m} \cos x_{i}\right)\right. \\
\left.\cdot\left(\prod_{m \geq k>j \geq 1}\left(\cos x_{j}-\cos x_{k}\right)\right)\right]\left.\right|_{x_{1}=x_{2}=\cdots=x_{m}=x} \\
=m !\left(\prod_{k=0}^{m-2} k !\right) \cos x \cdot \sin ^{m(m+1) / 2} x .
\end{gathered}
$$

In a more general formulation, using the same arguments as before, we get the identity 


$$
\begin{aligned}
& \left.\left(\prod_{l=2}^{m} \partial_{x_{l}}^{l-1}\right)\left[2^{m(m-1) / 2} \prod_{k=1}^{m} \sin x_{k} \cdot \mid \begin{array}{ccccccc}
1 & \cos x_{1} & \cdots & \cos ^{m-n-1} x_{1} & \cos ^{m-n+1} x_{1} & \cdots & \cos ^{m+n-1} x_{1} \\
\vdots & \vdots & \vdots & \vdots & \vdots & \vdots & \vdots \\
1 & \cos x_{m} & \cdots & \cos ^{m-n-1} x_{m} & \cos ^{m-n+1} x_{m} & \cdots & \cos ^{m+n-1} x_{m}
\end{array}\right]\right|_{x_{1}=x_{2}=\cdots=x_{m}=x} \\
& =c_{m n} \cdot \sin ^{m(m+1) / 2} x \cdot \cos ^{n(n+1) / 2} x
\end{aligned}
$$

with $c_{m n}$ defined by (25).

\section{Additional Comments}

(i) There is some similarity of (24), especially of the case $n=0$ with some addition formulas for elliptic functions obtained by Kiepert, Brioschi, Frobenius, and Stickelberger $[21,22]$ although we were not able to find a direct connection with their results. In particular, we do not know the compact expression for $W(\sigma(x), \sigma(2 x), \ldots, \sigma(n x))$, where $\sigma(x)$ is a Weierstrass sigma function.

(ii) Taking $f=e^{i k x}, k^{2}=E$ in the formula (64) for the solutions of (71) we see that the related function is $\psi(x, E)=P(x, k) \cdot e^{i k x}$ where $P(x, k)$ is a polynomial of $k$. In general the potentials allowing the solutions of this form are called Bargmann potentials. All Bargmann potentials might be considered as a degenerate case of the hyperelliptic finite gap periodic potentials [23]. In particular the DPT potentials (72) might be obtained from the general m-gap hyper elliptic potentials by closing the gaps to the points $\alpha,(2 \alpha)^{2}, \ldots,(d \alpha)^{2},(d+2)^{2} \alpha^{2}, \ldots(d+2 n)^{2} \alpha^{2}$.

(iii) Darboux [2] also found the integrable elliptic generalization of the DPT potentials also representing the 4parametric generalization of the Lamé equation. This elliptic Darboux potential has the following form:

$$
\begin{aligned}
u(x)= & \frac{m(m-1) k^{2} c n^{2} x}{d n^{2} x}+\frac{\alpha(\alpha-1) d n^{2} x}{c n^{2} x} \\
& +\frac{p(p-1)}{s n^{2} x}+n(n-1) k^{2} \operatorname{sn}^{2} x .
\end{aligned}
$$

In (97) $s n, c n$, and $d n$ are usual Jacobi elliptic functions of modulus $k$ and $m, \alpha, p, n$ are arbitrary nonnegative integers. In the case $m=\alpha=n=0$, this equation reduces to Lamé equation first integrated by Hermite. The potentials of the form (97) were rediscovered recently by Trebich and Verdier [24] as a special reduction of the general formula $[23,25,26]$ describing all finite-gap periodic or quasi periodic potentials together with explicit solutions of the related Schrödinger equation (Baker-Akhiezer functions). More general connections between the finite gap potentials and Fuchsian equations were recently discussed by Smirnov [27]. In the limit when one of the periods of the elliptic functions tends to infinity the Darboux-Verdier-Trebich potential reduces to the Darboux-Pöschl-Teller potential.

(iv) The DPT potentials and the Darboux-Verdier-Trebich potentials recently found some applications to the algebraic Bethe Ansatz construction for trigonometric case of the quantum Calogero-Moser multi particle system [28].

(v) Comparison of the results of Section 1 with [29-31] shows that the DPT trigonometric potentials produce the subfamily of two dimensional Huygens potentials $V(r, \varphi)$ via a formula

$$
V(r, \varphi)=\frac{1}{r^{2}}\left[\frac{m(m+1)}{\sin ^{2} \varphi}+\frac{n(n+1)}{\cos ^{2} \varphi}\right],
$$

since the generic 2D Huygens potentials in polar coordinates are described by the formula (in [29-31] the formula below was written in a slightly different but strictly equivalent form, using the wronskian of $m+1$ cosine functions):

$$
\begin{gathered}
V(r, \varphi)=-\frac{2}{r^{2}} \frac{\partial^{2}}{\partial \varphi^{2}} \ln W\left(\chi_{1}, \ldots, \chi_{m}\right), \\
\chi_{j}=\sin \left(k_{j} \varphi+\delta_{j}\right), \quad j=1, \ldots, m ; k_{j} \in \mathbb{Z}, \delta_{j} \in \mathbb{R} .
\end{gathered}
$$

Therefore, taking $k_{j}=a_{j}$, where $a_{j}$ are defined in (19) and taking $\delta_{j}=0$, we get the aforementioned link between the DPT potentials and Huygens potentials.

\section{Acknowledgment}

The authors wish to thank Dr. Y. Grandati for sending his preprint [13].

\section{References}

[1] G. Darboux, Sur une Proposition Relative aux Équations Linéaires, T. CXIV, Comptes rendus de l'Académie des Sciences, Serie I, Mathematics, Paris, France, 1882.

[2] G. Darboux, Sur une Équation linéaire, T. XCIV, Comptes Rendus de l'Académie des Sciences, Serie I, Mathematics, Paris, France, 1882.

[3] A. Treibich, "Difference analogs of elliptic KdV solitons and Schrödinger operators," International Mathematics Research Notices, no. 6, pp. 313-360, 2003. 
[4] A. Treibich and J.-L. Verdier, "Revêtements exceptionnels et sommes de 4 nombres triangulaires," Duke Mathematical Journal, vol. 68, no. 2, pp. 217-236, 1992.

[5] G. Pöschl and E. Teller, "Bemerkungen zur Quantenmechanik des anharmonischen Oszillators," Zeitschrift fur Physik A, vol. 83, pp. 143-151, 1933.

[6] M. Crum, “Associated Sturm-Liouville systems," The Quarterly Journal of Mathematics, vol. 6, pp. 121-127, 1955.

[7] V. B. Matveev, "Darboux transformation and the explicit solutions of differential-difference and difference-difference evolution equations I," Letters in Mathematical Physics, vol. 3, no. 3, pp. 213-216, 1979.

[8] V. B. Matveev, "Darboux transformations, covariance theorems and integrable systems," in L. D. Faddeev's Seminar on Mathematical Physics, vol. 201 of American Mathematical Society Translations, pp. 179-209, 2000.

[9] V. B. Matveev and M. A. Salle, Darboux Transformations and Solitons, Nonlinear Dynamics, Springer, Berlin, Germany, 1991.

[10] G. Darboux, Leçons sur la Théorie des Surfaces, vol. 2, Gauthier, Paris, France, 2nd edition, 1915.

[11] P. Gaillard and V. B. Matveev, Wronskian Addition Formula and Its Applications, vol. 161 of Max-Planck-Institut für Mathematik, MPI 02-31, 2002.

[12] P. Gaillard and V. B. Matveev, "Wronskian and Casorati determinant representations for Darboux-Pöschl-Teller potentials and their difference extensions," Journal of Physics A, vol. 42, no. 40, Article ID 404009, p. 16, 2009.

[13] Y. Grandati, "A short proof of the Gaillard-Matveev theorem based on shape invariance arguments," http://arxiv.org/ abs/1211.2392.

[14] V. B. Matveev, Functional-Difference Analogues of DarbouxPöschl-Teller Potentials, Max Planck Institut für Mathematik, Bonn, Germany, 2002.

[15] V. B. Matveev, "Functional-difference deformations of Darboux-Pöshl-Teller potentials," in Bilinear Integrable Systems: From Classical to Quantum, Continuous to Discrete, Nato Science Series, pp. 191-208, Springer, 2006.

[16] P. Gaillard, "A new family of deformations of Darboux-PöschlTeller potentials," Letters in Mathematical Physics, vol. 68, no. 2, pp. 77-90, 2004.

[17] P. Gaillard and V. B. Matveev, "New formulas for the eigenfunctions of the two-particle difference Calogero-Moser system," Letters in Mathematical Physics, vol. 89, no. 1, pp. 1-12, 2009.

[18] P. Gaillard and V. B. Matveev, "Casorati determinant formulation of the DDPTII equation and elementary Askey-Wilson functions," In press.

[19] G. Frobenius, "Über die determinante mehrerer functionen einer variabeln," Journal für die Reine und Angewandte Mathematik, vol. 77, pp. 245-257, 1874.

[20] S. G. Vlăduţ, "Isogeny class and Frobenius root statistics for abelian varieties over finite fields," Moscow Mathematical Journal, vol. 1, no. 1, pp. 125-139, 2001.

[21] G. Frobenius and L. Stickelberg, "Über die addition und Multiplication der elliptishen function," Journal fur die Reine und Angewandte Mathematik, vol. 88, pp. 146-184, 1880.

[22] E. T. Whittaker and G. N. Watson, A Course of Modern Analysis, Cambridge University Press, Cambridge, UK, 1996.

[23] E. D. Belokolos, A. I. Bobenko, A. R. Its, V. Z. Enolskij, and V. B. Matveev, Algebro-Geometric Approach to Nonlinear Integrable Equations, Springer Series in Nonlinear Dynamics, Springer, 1994.
[24] A. Treibich and J.-L. Verdier, "Revêtements tangentiels et sommes de 4 nombres triangulaires," Comptes Rendus de l'Académie des Sciences. Série I. Mathématique, vol. 311, no. 1, pp. 51-54, 1990.

[25] B. A. Dubrovin, V. B. Matveev, and S. P. Novikov, Non-Linear Equations of Korteweg-de Vries Type, Finite-Zone Linear Operators, and Abelian Varieties, vol. 60 of London Mathematical Society Lecture Note Series, Cambridge University Press, 1981.

[26] A. R. Its and V. B. Matveev, "Hill operators with a finite number of lacunae," Funktsional'nyi Analiz i ego Prilozheniia, vol. 9, no. 1, pp. 69-70, 1975.

[27] A. O. Smirnov, "Elliptic solutions and Heun's equation," http://arxiv.org/abs/math/0109149.

[28] K. Takemura, "On the eigenstates of the elliptic Calogero-Moser model," Letters in Mathematical Physics, vol. 53, no. 3, pp. 181194, 2000.

[29] Y. Berest, "Solution of a restricted Hadamard problem on Minkowski spaces," Communications on Pure and Applied Mathematics, vol. 50, no. 10, pp. 1019-1052, 1997.

[30] Y. Berest and I. M. Loutsenko, "Huygens' principle in Minkowski spaces and soliton solutions of the Korteweg-de Vries equation," Communications in Mathematical Physics, vol. 190, no. 1, pp. 113-132, 1997.

[31] Y. Berest, T. Cramer, and F. Eshmatov, "Heat kernel coefficients for two-dimensional Schrödinger operators," Communications in Mathematical Physics, vol. 283, no. 3, pp. 853-860, 2008. 


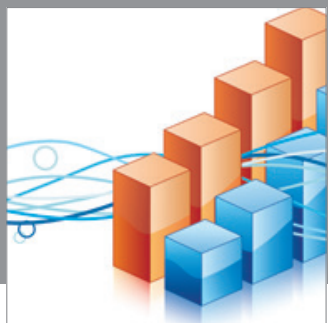

Advances in

Operations Research

mansans

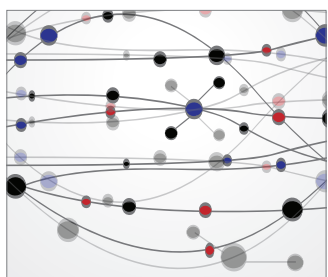

The Scientific World Journal
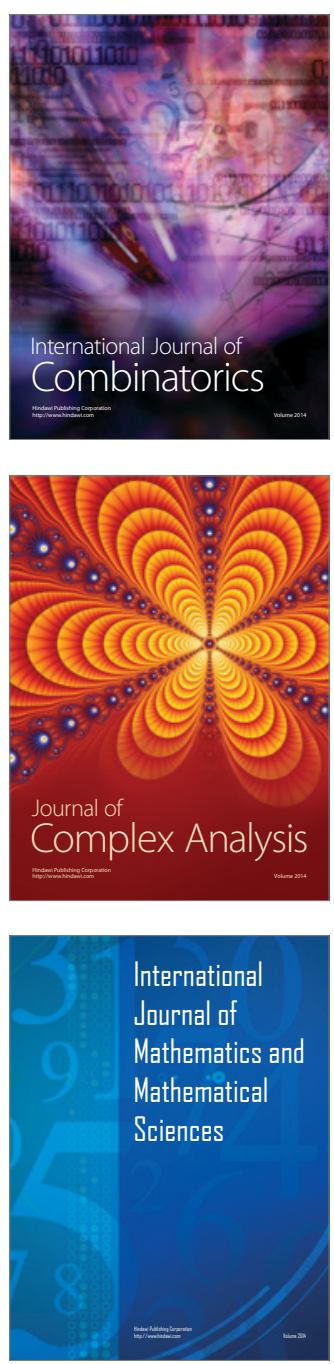
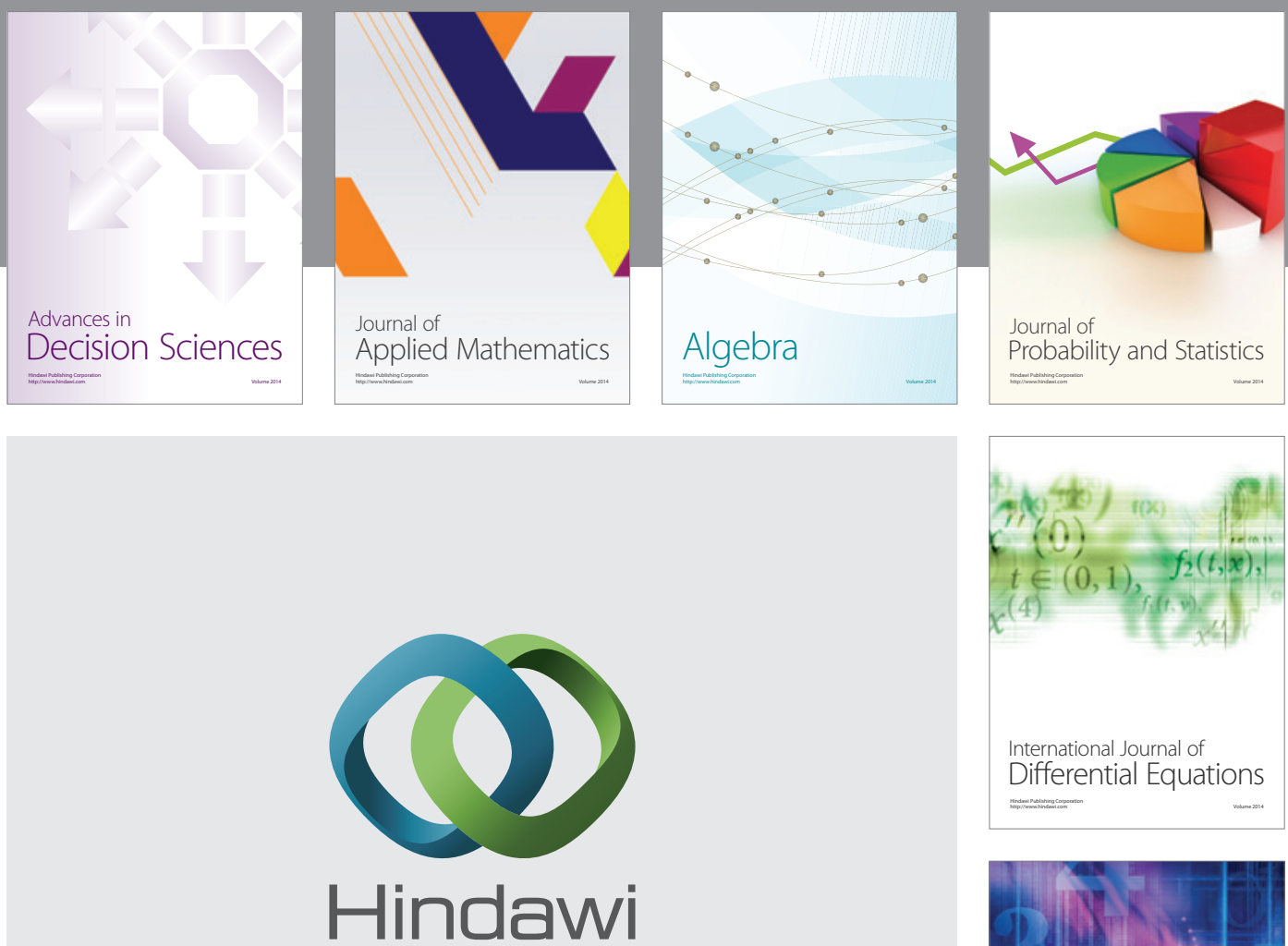

Submit your manuscripts at http://www.hindawi.com
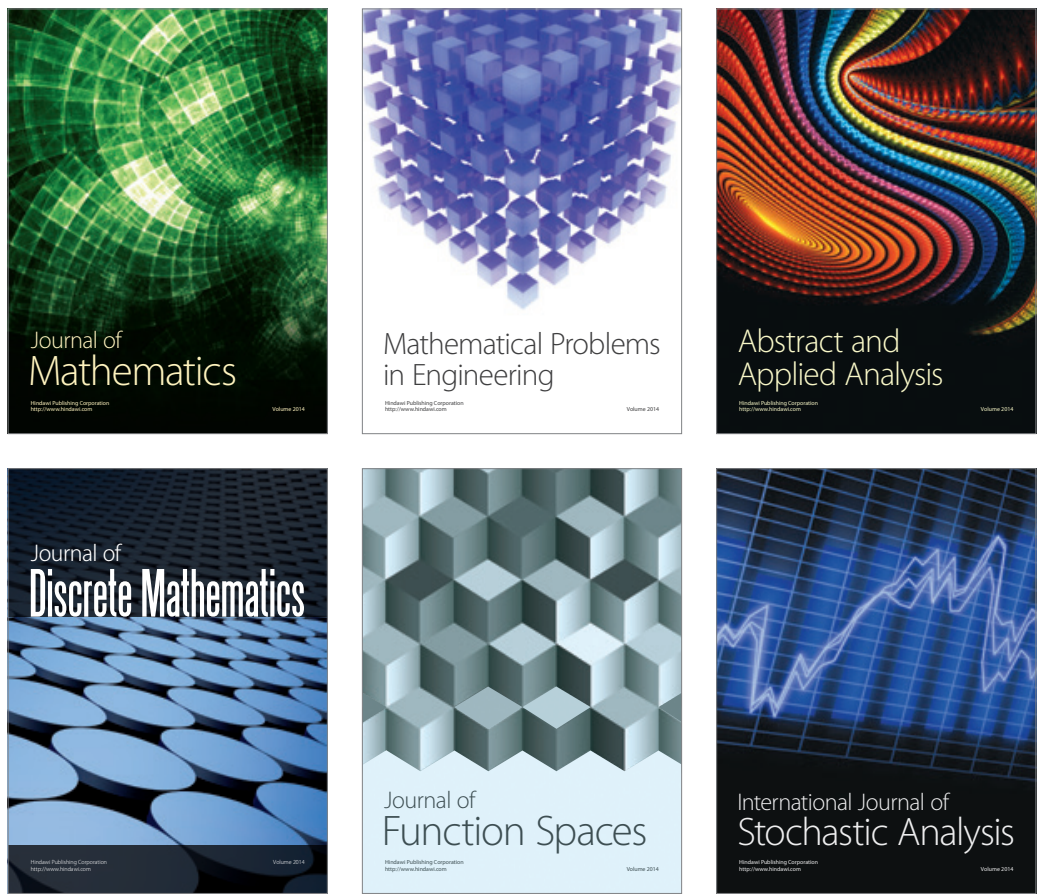

Journal of

Function Spaces

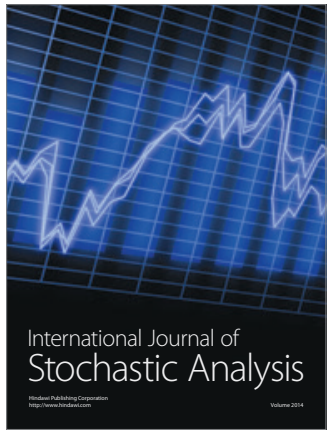

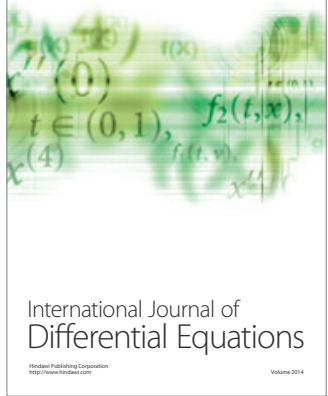
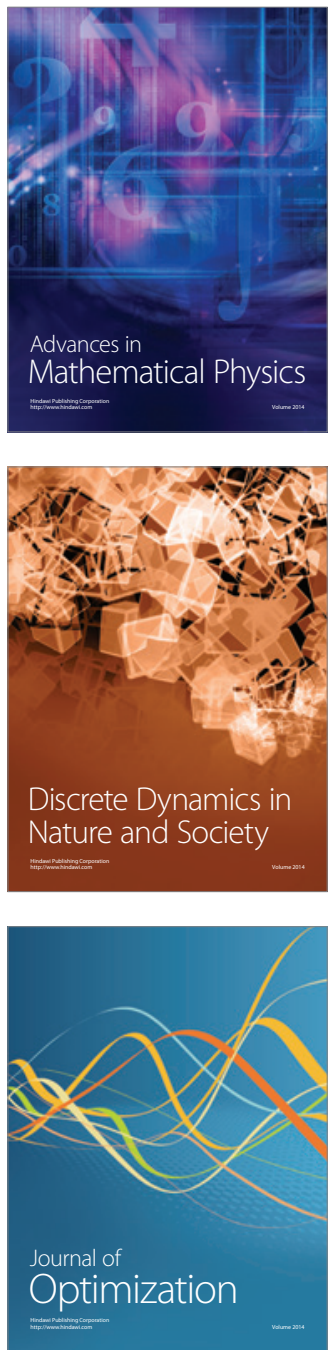